\title{
Projections and Reflections in Vector Space
}

\author{
Kung-Kuen Tse \\ Department of Mathematics, Kean University, Union, NJ, USA \\ Email: ktse@kean.edu
}

Received 4 April 2016; accepted 16 May 2016; published 19 May 2016

Copyright (C) 2016 by author and Scientific Research Publishing Inc.

This work is licensed under the Creative Commons Attribution International License (CC BY).

http://creativecommons.org/licenses/by/4.0/

c) (i) Open Access

\begin{abstract}
We study projections onto a subspace and reflections with respect to a subspace in an arbitrary vector space with an inner product. We give necessary and sufficient conditions for two such transformations to commute. We then generalize the result to affine subspaces and transformations.
\end{abstract}

\section{Keywords}

\section{Projection, Reflection, Commute, Inner Product, Affine Subspace}

\section{Introduction}

Two lines $\ell_{1}$ and $\ell_{2}$ in $\mathbb{R}^{2}$ are considered. When is the reflection over $\ell_{1}$ followed by the reflection over $\ell_{2}$ the same as the reflection over $\ell_{2}$ followed by the reflection over $\ell_{1}$ ? It is easy to see that it is the case if and only if $\ell_{1} \perp \ell_{2}$ or $\ell_{1}=\ell_{2}$.

When considering subspaces of $\mathbb{R}^{3}$, we can ask similar questions for lines, for planes or for the mixed case of one line and one plane. Instead of addressing those cases one by one, we generalize the situation of arbitrary two linear subspaces of a vector space with an inner product.

\section{Projection}

Supposing that $U$ is a vector space equipped with an inner product, $V \subset U$ is a linear subspace of $U$. Given a vector $u \in U$, we know from linear algebra [1] [2] that $u$ can be decomposed uniquely as $u=p_{V}(u)+u^{\prime}$ where $p_{V}(u) \in V$ is the projection of the vector $u$ onto $V$ and $u^{\prime} \perp V$, i.e. $U=V \oplus V^{\perp}$.

Here are some elementary properties of the projection $p_{V}$ :

1) $p_{V}$ is linear.

2) $u \in V$ if and only if $p_{V}(u)=u$.

3) $u \in V^{\perp}$ if and only if $p_{V}(u)=0$.

4) $V \cap W \subset p_{V}(W)$. 
5) If $V_{1}$ and $V_{2}$ are subspaces of $U$, then $p_{V_{1} \oplus V_{2}}(u)=p_{V_{1}}(u)+p_{V_{2}}(u)$, for all $u \in U$.

6) If $V_{1}, V_{2}$ and $W$ are subspaces of $U$, then $p_{V_{1} \oplus V_{2}}(W) \subset p_{V_{1}}(W) \oplus p_{V_{2}}(W)$.

7) If $V_{1}, V_{2}$ and $W$ are subspaces of $U$, then $p_{W}\left(V_{1} \oplus V_{2}\right)=p_{W}\left(V_{1}\right) \oplus p_{W}\left(V_{2}\right)$.

Lemma 2.1. Supposing that $U$ is a linear space and $V$, $W$ are two linear subspaces of $U$, if $p_{W}(V)=p_{V}(W)$ then $p_{W}(V)=p_{V}(W)=V \cap W$.

Proof. We first show that $p_{V}(W)=V \cap W$. Since $p_{V}(W) \subset V$ and $p_{V}(W)=p_{W}(V) \subset W$, we have $p_{V}(W) \subset V \cap W$. On the other hand, if $u \in V \cap W$, then $u \in V$, hence $u=p_{V}(u) \in p_{V}(W)$ and thus $V \cap W \subset p_{V}(W)$. As a result, $p_{V}(W)=V \cap W$. The proof of $p_{W}(V)=V \cap W$ is similar.

Suppose $U$ is a vector space and $V, W$ are two subspaces of $U$. Intersecting the identity $U=(V \cap W) \oplus$ $(V \cap W)^{\perp}$ with $V$ and $W$, we get $V=(V \cap W) \oplus\left(V \cap(V \cap W)^{\perp}\right)$ and $W=(V \cap W) \oplus\left(W \cap(V \cap W)^{\perp}\right)$. It is obvious that these two sums are orthogonal.

Denote $V^{\prime}=V \cap(V \cap W)^{\perp}$ and $W^{\prime}=W \cap(V \cap W)^{\perp}$. Using these notations, $V=(V \cap W) \oplus V^{\prime}$ and $W=(V \cap W) \oplus W^{\prime}$.

Lemma 2.2. $p_{V^{\prime}}\left(W^{\prime}\right)=p_{W^{\prime}}\left(V^{\prime}\right)=0$ if and only if $p_{W}(V)=p_{V}(W)$.

Poorf.

$$
\begin{aligned}
p_{V}(W) & =p_{(V \cap W) \oplus V^{\prime}}(W)=p_{(V \cap W) \oplus V^{\prime}}\left((V \cap W) \oplus W^{\prime}\right) \\
& \subset p_{V \cap W}(V \cap W) \oplus p_{V^{\prime}}(V \cap W) \oplus p_{V \cap W}\left(W^{\prime}\right) \oplus p_{V^{\prime}}\left(W^{\prime}\right) \\
& =(V \cap W) \oplus\{0\} \oplus\{0\} \oplus p_{V^{\prime}}\left(W^{\prime}\right) \\
& =(V \cap W) \oplus p_{V^{\prime}}\left(W^{\prime}\right)
\end{aligned}
$$

$(\Rightarrow)$ If $p_{V^{\prime}}\left(W^{\prime}\right)=0$, then $p_{V}(W) \subset V \cap W$. On the other hand, by the fourth property of projection above, $V \cap W \subset p_{V}(W)$. Similarly, $p_{W}(V)=V \cap W$. Thus, $p_{V}(W)=p_{W}(V)$.

$(\Leftarrow)$ By Lemma 2.1, $p_{V}(W)=V \cap W$. For $w^{\prime} \in W^{\prime}$,

$$
p_{V}\left(w^{\prime}\right)=p_{V \cap W}\left(w^{\prime}\right)+p_{V^{\prime}}\left(w^{\prime}\right)=p_{V^{\prime}}\left(w^{\prime}\right)
$$

$p_{V}\left(w^{\prime}\right) \in p_{V}(W)=V \cap W \quad$ and $\quad p_{V^{\prime}}\left(w^{\prime}\right) \in V^{\prime}$, but $(V \cap W) \cap V^{\prime}=0$, we must have $p_{V^{\prime}}\left(w^{\prime}\right)=0$, i.e. $p_{V^{\prime}}\left(W^{\prime}\right)=0$. Similarly, $p_{W^{\prime}}\left(V^{\prime}\right)=0$.

Theorem 2.3. Supposing that $U$ is a vector space and $V$, $W$ are two subspaces of $U$, then $p_{V} \circ p_{W}=p_{W} \circ p_{V}$ if and only if $p_{W}(V)=p_{V}(W)$.

Proof. $(\Rightarrow)$ Assume that $p_{W}\left(p_{V}(u)\right)=p_{V}\left(p_{W}(u)\right)$ for all $u \in U$. In particular,

$$
p_{W}(v)=p_{W}\left(p_{V}(v)\right)=p_{V}\left(p_{W}(v)\right) \in p_{V}(W) \text { for all } v \in V .
$$

Thus, $p_{W}(V) \subset p_{V}(W)$. Similarly, $p_{V}(W) \subset p_{W}(V)$.

$(\Leftarrow)$ Assume $p_{W}(V)=p_{V}(W)$. By Lemma 2.2, $\quad p_{V^{\prime}}\left(W^{\prime}\right)=p_{W^{\prime}}\left(V^{\prime}\right)=0$.

$$
\begin{aligned}
p_{W}\left(p_{V}(u)\right) & =p_{W}\left(p_{V \cap W}(u)+p_{V^{\prime}}(u)\right) \\
& =p_{W}\left(p_{V \cap W}(u)\right)+p_{W}\left(p_{V^{\prime}}(u)\right) \\
& =p_{W}\left(p_{V \cap W}(u)\right)+p_{V \cap W}\left(p_{V^{\prime}}(u)\right)+p_{W^{\prime}}\left(p_{V^{\prime}}(u)\right) \\
& =p_{V \cap W}(u) .
\end{aligned}
$$

Similarly, $p_{V}\left(p_{W}(u)\right)=p_{V \cap W}(u)$.

\section{Reflection over a Subspace}

Supposing that $U$ is a vector space equipped with an inner product, $V \subset U$ is a subspace of $U$. We define the refection of $u \in U$ with respect to $V$ as

$$
r_{V}(u)=2 p_{V}(u)-u .
$$

The above formula can be easily derived from the observation that $p_{V}(u)=\frac{1}{2}\left(u+r_{V}(u)\right)$. Note that if $u \in V$, then $r_{V}(u)=u$. 
Lemma 3.1. Supposing that $U$ is a vector space and $V, W$ are two vector subspaces of $U$, then $r_{V} \circ r_{W}=r_{W} \circ r_{V}$ if and only if $p_{V} \circ p_{W}=p_{W} \circ p_{V}$.

Proof.

$$
\begin{aligned}
r_{W}\left(r_{V}(u)\right) & =2 p_{W}\left(r_{V}(u)\right)-r_{V}(u)=2 p_{W}\left(2 p_{V}(u)-u\right)-\left(2 p_{V}(u)-u\right) \\
& =4 p_{W}\left(p_{V}(u)\right)-2 p_{W}(u)-2 p_{V}(u)+u .
\end{aligned}
$$

Similarly, $r_{V}\left(r_{W}(u)\right)=4 p_{V}\left(p_{W}(u)\right)-2 p_{V}(u)-2 p_{W}(u)+u$. Hence,

$$
r_{W} \circ r_{V}=r_{V} \circ r_{W} \text { if and only if } p_{W} \circ p_{V}=p_{V} \circ p_{W} \text {. }
$$

Theorem 3.2. Supposing that $U$ is a vector space and $V$, $W$ are two subspaces of $U$, then $r_{V} \circ r_{W}=r_{W} \circ r_{V}$ if and only if $p_{V}(W)=p_{W}(V)$.

Poor. By Lemma 3.1, $r_{V} \circ r_{W}$ if and only if $p_{V} \circ p_{W}=p_{W} \circ p_{V}$. By Theorem 2.3, $p_{V} \circ p_{W}=p_{W} \circ p_{V}$ if and only if $p_{V}(W)=p_{W}(V)$.

\section{Projection onto a Translated Subspace}

Define the projection of $u \in U$ onto a translated subspace $\hat{V}=V+v_{0}$ as

$$
p_{\hat{V}}(u)=p_{V}\left(u-v_{0}\right)+v_{0}=p_{V}(u)-p_{V}\left(v_{0}\right)+v_{0} .
$$

$p_{\hat{V}}$ is well defined: supposing $V+v_{0}=V+v_{0}^{\prime}$, then $v_{0}-v_{0}^{\prime} \in V$. Hence $p_{V}\left(v_{0}\right)-p_{V}\left(v_{0}^{\prime}\right)=p_{V}\left(v_{0}-v_{0}^{\prime}\right)=v_{0}-v_{0}^{\prime}$ and thus

$$
p_{V}(u)-p_{V}\left(v_{0}\right)+v_{0}=p_{V}(u)-p_{V}\left(v_{0}^{\prime}\right)+v_{0}^{\prime} .
$$

Theorem 4.1. $p_{\hat{V}} \circ p_{\hat{W}}=p_{\hat{W}} \circ p_{\hat{V}}$ if and only if $\hat{V} \cap \hat{W} \neq \phi$ and $p_{V}(W)=p_{W}(V)$.

Proof.

$$
\begin{aligned}
p_{\hat{V}}\left(p_{\hat{W}}(u)\right) & =p_{V}\left(p_{\hat{W}}(u)\right)-p_{V}\left(v_{0}\right)+v_{0} \\
& =p_{V}\left(p_{W}(u)-p_{W}\left(w_{0}\right)+w_{0}\right)-p_{V}\left(v_{0}\right)+v_{0} \\
& =p_{V}\left(p_{W}(u)\right)-p_{V}\left(p_{W}\left(w_{0}\right)\right)+p_{V}\left(w_{0}\right)-p_{V}\left(v_{0}\right)+v_{0} .
\end{aligned}
$$

Similarly, $p_{\hat{W}}\left(p_{\hat{V}}(u)\right)=p_{W}\left(p_{V}(u)\right)-p_{W}\left(p_{V}\left(v_{0}\right)\right)+p_{W}\left(v_{0}\right)-p_{W}\left(w_{0}\right)+w_{0}$.

Thus, $p_{\hat{V}}\left(p_{\hat{W}}(u)\right)=p_{\hat{W}}\left(p_{\hat{V}}(u)\right)$ if and only if

$$
\left\{\begin{array}{l}
p_{V}\left(p_{W}(u)\right)=p_{W}\left(p_{V}(u)\right) \\
-p_{V}\left(p_{W}\left(w_{0}\right)\right)+p_{V}\left(w_{0}\right)-p_{V}\left(v_{0}\right)+v_{0}=-p_{W}\left(p_{V}\left(v_{0}\right)\right)+p_{W}\left(v_{0}\right)-p_{W}\left(w_{0}\right)+w_{0}
\end{array}\right.
$$

$(\Rightarrow)$ By Theorem 2.3, the first equation implies $p_{V}(W)=p_{W}(V)$. The second equation simply means that $\hat{V} \cap \hat{W} \neq \phi$.

$(\Leftarrow)$ By Theorem 2.3, the first equation is satisifed. To show the second equation, since $\hat{V} \cap \hat{W} \neq \phi$, we have $\tilde{v}+v_{0}=\tilde{w}+w_{0}$, for some $\tilde{v} \in V$ and $\tilde{w} \in W$, or $v_{0}=\tilde{w}+w_{0}-\tilde{v}$ :

$$
\begin{aligned}
& -p_{W}\left(p_{V}\left(v_{0}\right)\right)+p_{W}\left(v_{0}\right)+p_{V}\left(v_{0}\right)-v_{0} \\
& =-p_{W}\left(p_{V}\left(\tilde{w}+w_{0}-\tilde{v}\right)\right)+p_{W}\left(\tilde{w}+w_{0}-\tilde{v}\right)+p_{V}\left(\tilde{w}+w_{0}-\tilde{v}\right)-\tilde{w}-w_{0}+\tilde{v} \\
& =-p_{V}(\tilde{w})-p_{W}\left(p_{V}\left(w_{0}\right)\right)+p_{W}(\tilde{v})+\tilde{w}+p_{W}\left(w_{0}\right)-p_{W}(\tilde{v})+p_{V}(\tilde{w})+p_{V}\left(w_{0}\right)-\tilde{v}-\tilde{w}-w_{0}+\tilde{v} \\
& =-p_{V}\left(p_{W}\left(w_{0}\right)\right)+p_{W}\left(w_{0}\right)+p_{V}\left(w_{0}\right)-w_{0}
\end{aligned}
$$

which is the second equation.

\section{Reflection over a Translated Subspace}

We next discuss the reflection over a translated subspace. Let $V \subset U$ be a subspace. A translated subspace is $\hat{V}=V+v_{0}$ for some $v_{0} \in U$. We define the reflection of $u \in U$ over $\hat{V}$ as 


$$
r_{\hat{V}}(u)=r_{V}\left(u-v_{0}\right)+v_{0}=r_{V}(u)-r_{V}\left(v_{0}\right)+v_{0} .
$$

$r_{\hat{V}}$ is well-defined: supposing $V+v_{0}=V+v_{0}^{\prime}$, then $v_{0}-v_{0}^{\prime} \in V$ and hence $r_{V}\left(v_{0}\right)-r_{V}\left(v_{0}^{\prime}\right)=r_{V}\left(v_{0}-v_{0}^{\prime}\right)=v_{0}-v_{0}^{\prime}$. As a result,

$$
r_{V}(u)-r_{V}\left(v_{0}\right)+v_{0}=r_{V}(u)-r_{V}\left(v_{0}^{\prime}\right)+v_{0}^{\prime} .
$$

Supposing $\hat{W}=W+w_{0}$ for some $w_{0} \in W$ is another translated subspace.

$$
\begin{aligned}
r_{\hat{W}}\left(r_{\hat{v}}(u)\right) & =r_{W}\left(r_{\hat{v}}(u)\right)-r_{W}\left(w_{0}\right)+w_{0} \\
& =r_{W}\left(r_{V}(u)-r_{V}\left(v_{0}\right)+v_{0}\right)-r_{W}\left(w_{0}\right)+w_{0} \\
& =r_{W}\left(r_{V}(u)\right)-r_{W}\left(r_{V}\left(v_{0}\right)\right)+r_{W}\left(v_{0}\right)-r_{W}\left(w_{0}\right)+w_{0} .
\end{aligned}
$$

Similarly, $r_{\hat{V}}\left(r_{\hat{W}}(u)\right)=r_{V}\left(r_{W}(u)\right)-r_{V}\left(r_{W}\left(w_{0}\right)\right)+r_{V}\left(w_{0}\right)-r_{V}\left(v_{0}\right)+v_{0}$.

Theorem 5.1. $r_{\hat{W}} \circ r_{\hat{V}}=r_{\hat{V}} \circ r_{\hat{W}}$ if and only if $\hat{V} \cap \hat{W} \neq \phi$ and $p_{V}(W)=p_{W}(V)$.

Proof. $r_{\hat{W}}\left(r_{\hat{v}}(u)\right)=r_{\hat{V}}\left(r_{\hat{W}}(u)\right)$ if and only if

$$
\left\{\begin{array}{l}
r_{W}\left(r_{V}(u)\right)=r_{V}\left(r_{W}(u)\right) \\
-p_{W}\left(p_{V}\left(v_{0}\right)\right)+p_{W}\left(v_{0}\right)-p_{W}\left(w_{0}\right)+w_{0}=-p_{V}\left(p_{W}\left(w_{0}\right)\right)+p_{V}\left(w_{0}\right)-p_{V}\left(v_{0}\right)+v_{0}
\end{array}\right.
$$

$(\Rightarrow)$ By Theorem 3.2, $r_{V} \circ r_{W}=r_{W} \circ r_{V}$ implies $p_{V}(W)=p_{W}(V)$. The second equation simply means $\hat{V} \cap \hat{W} \neq \phi$.

$(\Leftarrow)$ We express $r_{\hat{W}}\left(r_{\hat{v}}(u)\right)$ and $r_{\hat{V}}\left(r_{\hat{W}}(u)\right)$ in terms of projections:

$$
\begin{aligned}
& r_{\hat{W}}\left(r_{\hat{V}}(u)\right)=r_{W}\left(r_{V}(u)\right)-4 p_{W}\left(p_{V}\left(v_{0}\right)\right)+4 p_{W}\left(v_{0}\right)+2 p_{V}\left(v_{0}\right)-2 v_{0}-2 p_{W}\left(w_{0}\right)+2 w_{0}, \\
& r_{\hat{V}}\left(r_{\hat{W}}(u)\right)=r_{V}\left(r_{W}(u)\right)-4 p_{V}\left(p_{W}\left(w_{0}\right)\right)+4 p_{V}\left(w_{0}\right)+2 p_{W}\left(w_{0}\right)-2 w_{0}-2 p_{V}\left(v_{0}\right)+2 v_{0} .
\end{aligned}
$$

By Theorem 3.2, $p_{V}(W)=p_{W}(V)$ implies $r_{V} \circ r_{W}=r_{W} \circ r_{V}$. By Lemma 3.1, we also have $p_{V} \circ p_{W}=p_{W} \circ p_{V}$. To show $r_{\hat{W}} \circ r_{\hat{V}}=r_{\hat{V}} \circ r_{\hat{W}}$, it suffices to verify the second equation

$$
-p_{W}\left(p_{V}\left(v_{0}\right)\right)+p_{W}\left(v_{0}\right)+p_{V}\left(v_{0}\right)-v_{0}=-p_{V}\left(p_{W}\left(w_{0}\right)\right)+p_{V}\left(w_{0}\right)+p_{W}\left(w_{0}\right)-w_{0} .
$$

Since $\hat{V} \cap \hat{W} \neq \phi$, we must have $\tilde{v}+v_{0}=\tilde{w}+w_{0}$ for some $\tilde{v} \in V$ and $\tilde{w} \in W$, or $v_{0}=\tilde{w}+w_{0}-\tilde{v}$ :

$$
\begin{aligned}
- & p_{W}\left(p_{V}\left(v_{0}\right)\right)+p_{W}\left(v_{0}\right)+p_{V}\left(v_{0}\right)-v_{0} \\
= & -p_{W}\left(p_{V}\left(\tilde{w}+w_{0}-\tilde{v}\right)\right)+p_{W}\left(\tilde{w}+w_{0}-\tilde{v}\right)+p_{V}\left(\tilde{w}+w_{0}-\tilde{v}\right)-\tilde{w}-w_{0}+\tilde{v} \\
= & -p_{W}\left(p_{V}(\tilde{w})\right)-p_{W}\left(p_{V}\left(w_{0}\right)\right)+p_{W}(\tilde{v})+\tilde{w}+p_{W}\left(w_{0}\right)-p_{W}(\tilde{v}) \\
& +p_{V}(\tilde{w})+p_{V}\left(w_{0}\right)-\tilde{v}-\tilde{w}-w_{0}+\tilde{v} \\
= & -p_{W}\left(p_{V}(\tilde{w})\right)-p_{W}\left(p_{V}\left(w_{0}\right)\right)+p_{W}\left(w_{0}\right)+p_{V}(\tilde{w})+p_{V}\left(w_{0}\right)-w_{0} \\
= & -p_{V}\left(p_{W}(\tilde{w})\right)-p_{V}\left(p_{W}\left(w_{0}\right)\right)+p_{W}\left(w_{0}\right)+p_{V}(\tilde{w})+p_{V}\left(w_{0}\right)-w_{0} \\
= & -p_{V}(\tilde{w})-p_{V}\left(p_{W}\left(w_{0}\right)\right)+p_{W}\left(w_{0}\right)+p_{V}(\tilde{w})+p_{V}\left(w_{0}\right)-w_{0} \\
= & -p_{V}\left(p_{W}\left(w_{0}\right)\right)+p_{V}\left(w_{0}\right)+p_{W}\left(w_{0}\right)-w_{0}
\end{aligned}
$$

\section{Mixed Transformations}

Theorem 6.1. $\quad p_{\hat{V}} \circ r_{\hat{W}}=r_{\hat{W}} \circ p_{\hat{V}}$ if and only if $\hat{V} \cap \hat{W} \neq \phi$ and $p_{V}(W)=p_{W}(V)$.

Theorem 6.2. $\quad p_{\hat{V}} \circ r_{\hat{W}}=r_{\hat{V}} \circ p_{\hat{W}}$ if and only if $\hat{V} \cap \hat{W} \neq \phi$ and $V=W$.

Theorem 6.3. $\quad p_{\hat{V}} \circ r_{\hat{W}}=p_{\hat{W}} \circ r_{\hat{V}}$ if and only if $\hat{V} \cap \hat{W} \neq \phi$ and $V=W$.

\section{Generalizations}

If we denote $\Sigma_{n}$, the permutation group of order $n$, then 
Theorem 7.1.

$$
p_{V_{\sigma(1)}} \circ \cdots \circ p_{V_{\sigma(n)}}=p_{V_{\tau(1)}} \circ \cdots \circ p_{V_{\tau(n)}} \text { for all } \sigma, \tau \in \Sigma_{n}
$$

if and only if

$$
p_{V_{\sigma(1)}} \cdots p_{V_{\sigma(n-1)}}\left(V_{\sigma(n)}\right)=p_{V_{\tau(1)}} \cdots p_{V_{\tau(n-1)}}\left(V_{\tau(n)}\right) \text { for all } \sigma, \tau \in \Sigma_{n} \text {. }
$$

Theorem 7.2.

$$
r_{V_{\sigma(1)}} \circ \cdots \circ r_{V_{\sigma(n)}}=r_{V_{\tau(1)}} \circ \cdots \circ r_{V_{\tau(n)}} \text { for all } \sigma, \tau \in \Sigma_{n}
$$

if and only if

$$
p_{V_{\sigma(1)}} \cdots p_{V_{\sigma(n-1)}}\left(V_{\sigma(n)}\right)=p_{V_{\tau(1)}} \cdots p_{V_{\tau(n-1)}}\left(V_{\tau(n)}\right) \text { for all } \sigma, \tau \in \Sigma_{n} \text {. }
$$

Theorem 7.3.

$$
p_{\hat{V}_{\sigma(1)}} \circ \cdots \circ p_{\hat{V}_{\sigma(n)}}=p_{\hat{V}_{\tau(1)}} \circ \cdots \circ p_{\hat{V}_{\tau(n)}} \text { for all } \sigma, \tau \in \Sigma_{n}
$$

if and only if $\hat{V}_{i} \cap \hat{V}_{j} \neq \phi$ for all $i, j$ and

$$
p_{V_{\sigma(1)}} \cdots p_{V_{\sigma(n-1)}}\left(V_{\sigma(n)}\right)=p_{V_{\tau(1)}} \cdots p_{V_{\tau(n-1)}}\left(V_{\tau(n)}\right) \text { for all } \sigma, \tau \in \Sigma_{n} .
$$

Theorem 7.4.

$$
r_{\hat{V}_{\sigma(1)}} \circ \cdots \circ r_{\hat{V}_{\sigma(n)}}=r_{\hat{v}_{\tau(1)}} \circ \cdots \circ r_{\hat{V}_{\tau(n)}} \text { for all } \sigma, \tau \in \Sigma_{n}
$$

if and only if $\hat{V}_{i} \cap \hat{V}_{j} \neq \phi$ for all $i, j$ and

$$
p_{V_{\sigma(1)}} \cdots p_{V_{\sigma(n-1)}}\left(V_{\sigma(n)}\right)=p_{V_{\tau(1)}} \cdots p_{V_{\tau(n-1)}}\left(V_{\tau(n)}\right) \text { for all } \sigma, \tau \in \Sigma_{n} .
$$

\section{References}

[1] Lay, D. (2011) Linear Algebra and Its Applications. 4th Edition, Pearson, USA.

[2] Strang, G. (2005) Linear Algebra and Its Applications. 4th Edition, Brooks Cole, USA. 